American Journal of Applied Sciences 8 (10): 945-952, 2011

ISSN 1546-9239

(C) 2011 Science Publications

\title{
Anticancer Activity of Some New Synthesized Tetrahydroquinoline and Tetrahydrochromene Carbonitrile Derivatives
}

\author{
${ }^{1}$ Usama W. Hawas, ${ }^{2}$ Mohamed A. Al-Omar, ${ }^{1,3}$ Abd El-Galil E. Amr \\ and ${ }^{1}$ Abu El-Ftouh G. Hammam \\ ${ }^{1}$ Department Applied Organic Chemistry, \\ National Research Center, Dokki, Cairo, Egypt \\ ${ }^{2}$ Department of Pharmaceutical Chemistry, College of Pharmacy, \\ King Saud University, Riyadh 11451, Saudi Arabia \\ ${ }^{3}$ Drug Exploration \& Development Chair (DEDC), College of Pharmacy, \\ King Saud University, Riyadh 11451, Saudi Arabia
}

\begin{abstract}
Problem statement: In continuation to our search for new heterocyclic chemistry based anticancer, the suggestion, synthesis, structure elucidation of some naphthalene, chromene and quinoline derivatives 3-7 were realized herein using 3-methylcyclohexanone 1 as a starting material. Approach: The antitumor activities of the newly synthesized compounds 4-7 were evaluated utilizing 60 different human tumor cell lines, representing leukemia, melanoma, lung, colon, brain, ovary, breast and prostate as well as kidney. Results: Some of the tested compounds exhibited better in vitro antitumor activities at low concentration $\left(\log 10 \mathrm{GI}_{50}=-4.7\right)$ against the used human tumor cell lines. Conclusion: From the obtained results, we can conclude that cyanopyridine and pyrane moieties fused to 3-methycyclohexane ring are essential for antitumor activities. In the present work, we can suggest that the anticancer activity is due to the presence of nitrogen heterocyclic rings and the presence of the nitrile groups $(\mathrm{CN})$ generally enhancing the activity.
\end{abstract}

Key words: Synthesis, REACTIONS, 2,6-Bis-(3,4-dimethoxybenzylidene)-3-methylcyclo-hexanone, tetrahydroquinoline, tetrahydrochromene, pyranes, cyanopyridine, anticancer activity

\section{INTRODUCTION}

The pyridine scaffold is a widespread structural motif that can be found in many natural products and in several pharmacologically interesting compounds. Therefore the synthesis of pyridine derivatives, aiming to develop new drugs, is an active research area. Therefore, cyanopyridyl derivatives are a potent inhibitor of dihydrouracil dehydrogenase and its coadministration with 1-ethoxymethyl-5-fluorouracil enhances the antitumor effect (Cocco et al., 2005). Dicyanopyridines derivatives have been described as intermediates in the synthesis of pyrido[2,3d]pyrimidines as antihistaminic agents (Quintela et al., 1997), pyridothieno-and pyridodithienotriazines endowed with antihistaminic and cytotoxic activity (Quintela et al., 1998), triazabenz[d,e]anthracene and tetrazabenz[d,e]anthracene that are DNA intercalating agents (Quintela and Peinador, 1996) and acyclo-3deazapyrimidine S-nucleosides that are active toward
HIV (Attia and Ismail, 2003). In a previous work we reported that certain of our newly substituted heterocyclic compounds exhibited antitumor (Amr et al., 2006; Hammam et al., 2003; 2001, Velusamy and Palaniappan, 2011; Covyeou et al., 2011), antiparkinsonian (Amr et al., 2003a), antimicrobial (Amr et al., 2003b; Attia et al., 2000) and antiinflammatory (Abou-Ghalia et al., 2003) activities. Heterocyclic derivatives present an interesting group of compounds many of which possess widespread pharmacological properties (Borgio et al., 2011; Ngoy et al., 2011), specially as anticancer, analgesic, antipyretic and antirheumatic activities (Mohamed et al., 2010; Bryzgalov et al., 2006; Rezvani and Shariati, 2010). In addition, the pharmacological and antitumor activities of many compounds containing heterocyclic ring have been reviewed (Amr, 2000; Hammam et al., 2000, Mohamed et al., 2011). Also, the heterocyclic nitrogen derivatives exhibited a general ionophoric potency for divalent cations (Hassan et al., 2003a) and

Corresponding Author: Mohamed A. Al-Omar, Department of Pharmaceutical Chemistry, College of Pharmacy,

King Saud University, Riyadh 11451, Saudi Arabia 
used a novel thiocyanate-selective membrane sensor (Hassan et al., 2003b). In view of these reports and in continuation of our previous works in heterocyclic chemistry; we have herein synthesized some new heterocyclic ring fused with substituted cyclohexene structure for the evaluation of their anticancer activity.

\section{MATERIALS AND METHODS}

Chemistry: All melting points were determined on open glass capillaries using an Electrothermal IA 9000 digital melting point apparatus. Elemental analyses were performed on Elementar, Vario EL, Microanalytical Unit, National Research Centre, Cairo Egypt and were found within $\pm 0.4 \%$ of the theoretical values. Infrared (IR) spectra were recorded on Carlzeise Spectrophotometer model "UR 10" spectrophotometer using the $\mathrm{KBr}$ disc technique. ${ }^{1} \mathrm{H}-\mathrm{NMR}$ spectra were recorded on Varian Gemini $270 \mathrm{MHz}$ spectrometer (DMSO- $\left.\mathrm{d}_{6}\right),{ }^{13} \mathrm{C}-\mathrm{NMR}$ spectra were recorded on Varian Gemini $67.5 \mathrm{MHz}$ spectrometer and the chemical shifts are given in $\delta(\mathrm{ppm})$ downfield from Tetramethylsilane (TMS) as an internal standard. The mass spectra (MS) were measured using a Finnigan SSQ 7000 mass spectrometer. Follow up of the reactions and checking the purity of the compounds were made by TLC on silica gel-precoated aluminum sheets (Type $60 \mathrm{~F}_{254}$, Merck, Darmstadt, Germany) and the spots were detected by exposure to UV lamp at $\lambda_{254} \mathrm{~nm}$ for few seconds. The anticancer screening occurred in United States National Institute of Health (NIH)/National Cancer Institute (NCI).

Synthesis of 3-amino-2,4-dicyano-4-(3-methylcyclohexylidene)-butyramide-2-ene (3): A mixture of 1 $(1.12 \mathrm{~g}, 10 \mathrm{mmol})$, malononitrile $(0.66 \mathrm{~g}, 10 \mathrm{mmol})$ and $\beta$-alanine $(50 \mathrm{mg})$ in absolute ethanol $50 \mathrm{ml}$ was refluxed for $48 \mathrm{~h}$. The reaction mixture was evaporated under reduced pressure, the obtained residue was dissolved in water/3N HCl $(100 \mathrm{~mL}, 1: 1)$. The product was extracted with diethyl ether, the ethereal solution was dried over anhydrous sodium sulphate. The ethereal solution was evaporated under reduced pressure, crystallized from ethanol to give the title product 3. Yield $55 \%, \mathrm{mp} 230^{\circ} \mathrm{C}$; IR $\left(\mathrm{KBr}, \mathrm{cm}^{-1}\right): 3450$, 3200, 2210, 1710; ${ }^{1} \mathrm{H}$ NMR: 9.23 (s, 2H, $\mathrm{NH}_{2}$, exchangeable with $\left.\mathrm{D}_{2} \mathrm{O}\right), 5.15(\mathrm{~s}, 1 \mathrm{H}, \mathrm{CH}), 4.82(\mathrm{t}, 2 \mathrm{H}$, $\mathrm{NH}_{2}$, exchangeable with $\left.\mathrm{D}_{2} \mathrm{O}\right), 2.13-1.16(3 \mathrm{~m}, 9 \mathrm{H}$, cyclohexene protons), 0.98 (d, $\left.3 \mathrm{H}, \mathrm{CH}_{3}\right) ; \mathrm{MS}(\mathrm{EI}): \mathrm{m} / \mathrm{z}$ $219\left[\mathrm{M}^{+}\right](13), 175\left[\mathrm{M}^{+}-\mathrm{CONH}_{2}\right](100), 160\left[175-\mathrm{CH}_{3}\right]$,
132 [160-CO]. Anal. Calcd. for $\mathrm{C}_{12} \mathrm{H}_{17} \mathrm{~N}_{3} \mathrm{O}$ : C, 65.73; H, 7.81; N, 19.16\%. Found: C, C, 65.64; H, 7.75; N, $19.05 \%$.

Synthesis of 2-amino-4-aryl-5-methyl-5,6,7,8tetrahydronaphtho-1,3-dicarbonitrile (4a-c): Method A: To a mixture of compound $3(1.22 \mathrm{~g}, 5$ $\mathrm{mmol}$ ) and aromatic aldehyde, namely, p-chlorobenzaldehyde, or 3,4-dimethoxybenzaldehyde or 3,4,5trimethoxybenzaldehyde $(5 \mathrm{mmol})$ in ethanol $50 \mathrm{ml}$, potassium hydroxide $0.4 \mathrm{~g}$ in $5 \mathrm{ml}$ water was added. The reaction mixture was stirred at room temperature for $4 \mathrm{~h}$, the solvent was concentrated under reduced pressure and the obtained solid was filtered off, washed with water, dried and crystallized from the proper solvent to give the title compounds $4 \mathrm{a}-\mathrm{c}$.

Method B: To a mixture of $1(1.12 \mathrm{~g}, 10 \mathrm{mmol})$, aromatic aldehyde, namely, p-chlorobenzaldehyde, or 3,4-dimethoxybenzaldehyde or 3,4,5-trimethoxybenzaldehyde $(10 \mathrm{mmol})$ and malononitrile $(20 \mathrm{mmol})$ in ethanol $50 \mathrm{ml}$, potassium hydroxide $0.4 \mathrm{~g}$ was added. The reaction mixture was refluxed for $6 \mathrm{~h}$. and concentrated under reduced pressure, the obtained solid was filtered off, washed with water, dried and crystallized from acetic acid/water to give the title compounds $4 \mathrm{a}-\mathrm{c}$.

2-Amino-4-(p-chlorophenyl)-5-methyl-5,6,7,8tetrahydronaphtho-1,3-dicarbonitrile (4a): Yield $75 \%$ [A], $76 \%$ [B], mp $210^{\circ} \mathrm{C}$; IR $\left(\mathrm{KBr}, \mathrm{cm}^{-1}\right): 3480$, 3200, 2216, 2212. ${ }^{1} \mathrm{H}-\mathrm{NMR}$ : 7.74-7.13 (m, 4H, Ar-H), $6.20\left(\mathrm{~s}, 2 \mathrm{H}, \mathrm{NH}_{2}\right.$, exchangeable with $\left.\mathrm{D}_{2} \mathrm{O}\right), 3.52(\mathrm{~m}$, $1 \mathrm{H}, \mathrm{CH}-5), 2.70-2.00(\mathrm{~m}, 6 \mathrm{H}$, cyclohexene protons); 1.18 (d, 3H, 5- $\mathrm{CH}_{3}$ ); $\mathrm{MS}$ (EI): m/z $321\left[\mathrm{M}^{+}\right]$(100), 244 $\left[\mathrm{M}^{+}-\mathrm{CN}, \mathrm{NH}_{2}, \mathrm{Cl}\right]$ and 190 [244-CN, $\left.\mathrm{CH}-\mathrm{CH}_{3}\right]$. Anal. Calcd. for $\mathrm{C}_{19} \mathrm{H}_{16} \mathrm{ClN}_{3}: \mathrm{C}, 70.91 ; \mathrm{H}, 5.01 ; \mathrm{Cl}, 11.02, \mathrm{~N}$, 13.05\%. Found: $\mathrm{C}, 70.89 ; \mathrm{H}, 4.96 ; \mathrm{Cl}, 10.96, \mathrm{~N}$, $13.02 \%$.

2-Amino-4-(3,4-dimethoxy-phenyl)-5-methyl-5,6,7,8tetrahydronaphtho-1,3-dicarbonitrile (4b): Yield $70 \%$ [A], 65\% [B]; mp $191^{\circ} \mathrm{C}$; IR $\left(\mathrm{KBr}, \mathrm{cm}^{-1}\right): 3500$, 3200, 2222, 2216; ${ }^{1} \mathrm{H}-\mathrm{NMR}: 7.11$ (s, 1H, Ar-H), 6.85 $(\mathrm{d}, \mathrm{J}=8 \mathrm{~Hz}, 1 \mathrm{H}, \mathrm{Ar}-\mathrm{H}), 6.74(\mathrm{~d}, \mathrm{~J}=8 \mathrm{~Hz}, 1 \mathrm{H}, \mathrm{Ar}-\mathrm{H})$, $6.62\left(\mathrm{~s}, 2 \mathrm{H}, \mathrm{NH}_{2}\right.$, exchangeable with $\left.\mathrm{D}_{2} \mathrm{O}\right), 3.93,3.86$ $\left(2 \mathrm{~s}, 6 \mathrm{H}, 2 \mathrm{OCH}_{3}\right), 3.48(\mathrm{~m}, 1 \mathrm{H}, \mathrm{H}-5), 2.70-2.00(\mathrm{~m}, 6 \mathrm{H}$, cyclohexene protons); 1.18 (d, 3H, 5- $\left.\mathrm{CH}_{3}\right)$; MS (EI): $\mathrm{m} / \mathrm{z} 347\left[\mathrm{M}^{+}\right]$(100), $316\left[\mathrm{M}^{+}-\mathrm{OCH}_{3}\right], 272$ [316-CN, $\mathrm{NH}_{2}, 2 \mathrm{H}$ ] and 231 [272-CN, $\mathrm{CH}_{2}$ ]. Anal. Calcd. for $\mathrm{C}_{21} \mathrm{H}_{21} \mathrm{~N}_{3} \mathrm{O}_{2}$ : C, 72.59; H, 6.09; N, 12.09\%. Found: C, 72.55; H, 6.04; N, $12.04 \%$.

\section{2-Amino-4-(3,4,5-trimethoxy-phenyl)-5-methyl- 5,6,7,8-tetrahydronaphtho-1,3-dicarbonitrile}

(4c): 946 
Yield 65\% [A], 60\% [B], mp 250248C; IR $\left(\mathrm{KBr}, \mathrm{cm}^{-1}\right)$ : 3400, 3200, 2218, 2200; ${ }^{1} \mathrm{H}$ NMR: 6.40, $6.30(2 \mathrm{~s}, 2 \mathrm{H}$, Ar- $\mathrm{H}), 5.40\left(\mathrm{~s}, 2 \mathrm{H}, \mathrm{NH}_{2}\right.$, exchangeable with $\left.\mathrm{D}_{2} \mathrm{O}\right), 3.93$, $3.86,3.72\left(3 \mathrm{~s}, 9 \mathrm{H}, 3 \mathrm{OCH}_{3}\right), 3.41(\mathrm{~m}, 1 \mathrm{H}, \mathrm{CH}-5), 2.52-$ $1.46(\mathrm{~m}, 6 \mathrm{H}$, cyclohexene protons), $1.22(\mathrm{~d}, 3 \mathrm{H}, 5-$ $\left.\mathrm{CH}_{3}\right) ;{ }^{13} \mathrm{C}$-NMR: $153.9,153.8,150.3,150.2,96.7,96.7$, $147.2,132.8,126.2,115.9,115.7,105.8,61.1,56.4$, 38.0, 30.8, 28.2, 27.2, 21.6; MS (EI): $\mathrm{m} / \mathrm{z} 377\left[\mathrm{M}^{+}\right]$ (100), $345\left[\mathrm{M}^{+}-\mathrm{NH}_{2}, \mathrm{CH}_{3}\right], 151$ [345-CN, H]. Anal. Calcd. For $\mathrm{C}_{22} \mathrm{H}_{23} \mathrm{~N}_{3} \mathrm{O}_{3}$ : C, 70.00; H, 6.14; N, $11.13 \%$. Found: C, 70.03; H, 6.11; N, 11.10\%.

Synthesis of 2,6-bisarylmethelene-3-methylcyclohexanones (5a-c). To a mixture of 3-methylcyclohexanone 1 ( 0.01 mole) and the appropriate aromatic aldehyde, namely, p-chloro-, or 3,4-dimethoxy- or 3,4,5-trimethoxybenzaldehyde $(0.02 \mathrm{~mole})$ in $50 \mathrm{ml}$ ethanol, $\mathrm{KOH}\left(0.01\right.$ mole in $\left.5 \mathrm{ml} \mathrm{H}_{2} \mathrm{O}\right)$ was added. The reaction mixture was stirred at room temperature for 2 $\mathrm{hr}$, the yellow solid formed was collected by filtration and crystallized from the proper solvent to give compounds 5a-c, respectively.

2,6-Bis-(p-chlorobenzylidene)-3-methylcyclohexanone (5a): (Grever et al., 1992; Abdel-Latif and Abdallah, 2010).

2,6-Bis-(3,4-dimethoxybenzylidene)-3methylcyclohexanone (5b): (Boyd and Paull, 1995). 2,6-Bis-(3,4-dimethoxybenzylidene)-3methylcyclohexanone (5c): Yield $95 \%, \mathrm{mp} 159^{\circ} \mathrm{C}$ (EtOH); IR $\left(\mathrm{KBr}, \mathrm{cm}^{-1}\right)$ : 1680, 1660; ${ }^{1} \mathrm{H}$ NMR: 7.72 and $7.65(2 \mathrm{~s}, 2 \mathrm{H}$, benzylic proton), 7.54 and $7.33(2 \mathrm{~s}$, $4 \mathrm{H}, \mathrm{Ar}-\mathrm{H}), 3.94,3.83,3.75,3.61\left(4 \mathrm{~s}, 18 \mathrm{H}, 6 \mathrm{OCH}_{3}\right)$, 3.28 (b, 1H, CH-5), 1.92-1.54 (m, 4H, cyclohexene protons), 1.24 (d, 3H, 5- $\left.\mathrm{CH}_{3}\right)$; MS (EI): $\mathrm{m} / \mathrm{z} 468\left[\mathrm{M}^{+}\right]$ (100), $453\left[\mathrm{M}^{+}-\mathrm{CH}_{3}\right](65), 285\left[453-\mathrm{Ph}\left(\mathrm{OCH}_{3}\right)_{3}, \mathrm{H}\right]$ (54). Anal. Calcd. for $\mathrm{C}_{27} \mathrm{H}_{32} \mathrm{O}_{7}: \mathrm{C}, 69.21 ; \mathrm{H}, 6.88 \%$. Found: C, 69.18; H, 6.84\%.

Synthesis of 2-amino-8-(substituted-benzylidene)-4aryl-5-methyl-5,6,7,8-tetrahydro-4H-chromene-3-

carbonitrile (6a-c): To a mixture of compound 5a-c (5 $\mathrm{mmol})$, malononitrile $(0.33 \mathrm{~g}, 5 \mathrm{mmol})$ and sodium acetate anhydrous $(40 \mathrm{mmol})$ in $100 \mathrm{ml}$ ethanol, few drops of triethylamine were added. The reaction mixture was refluxed for $4 \mathrm{~h}$., after cooling, it was poured onto water with stirring, the obtained precipitate was collected by filtration, washed with water, dried and crystallized from the proper solvent to give $6 \mathrm{a}-\mathrm{c}$, respectively.

2-Amino-8-(p-chlorobenzylidene)-4-(pchlorophenyl)-5-methyl-5,6,7,8-tetrahydro-4Hchromene-3-carbonitrile (6a): Yield $60 \%, \mathrm{mp} 215^{\circ} \mathrm{C}$ $(\mathrm{EtOH})$; IR $\left(\mathrm{KBr}, \mathrm{cm}^{-1}\right): 3460,3310,2200$; ${ }^{1} \mathrm{H}$ NMR:
8.23-8.14 (m, 4H, Ar-H), 7.50-7.21 (m, 5H, Ar-H + benzylic proton), $6.80\left(\mathrm{~s}, 2 \mathrm{H}, \mathrm{NH}_{2}\right.$, exchangeable with $\left.\mathrm{D}_{2} \mathrm{O}\right), 4.15(\mathrm{~s}, 1 \mathrm{H}$, pyran-H), $3.32(\mathrm{~b}, 1 \mathrm{H}, \mathrm{CH}-5), 2.50-$ $1.50\left(\mathrm{~m}, 2 \mathrm{H}, 2 \mathrm{CH}_{2}\right.$ of cyclohexene ring); $1.17(\mathrm{~d}, 3 \mathrm{H}, 5-$ $\mathrm{CH}_{3}$ ). MS (EI): m/z $423\left[\mathrm{M}^{+}\right](100), 311\left[\mathrm{M}^{+}-(\mathrm{p}-\right.$ chlorophenyl)] (85). Anal. Calcd. for $\mathrm{C}_{24} \mathrm{H}_{20} \mathrm{~N}_{2} \mathrm{OCl}_{2}$ : C, 68.09; H, 4.76; Cl, 16.75; N, 6.62\%. Found: C, 68.00; $\mathrm{H}, 4.70 ; \mathrm{Cl}, 16.69 ; \mathrm{N}, 6.55 \%$.

2-Amino-8-(3,4-dimethoxy-benzylidene)-4-(3,4dimethoxy-phenyl)-5-methyl-5,6,7,8-tetra-hydro-4Hchromene-3-carbonitrile (6b): Yield $85 \%, \mathrm{mp} 212^{\circ} \mathrm{C}$ (EtOH); IR (KBr, cm $\left.{ }^{-1}\right): 3450,3300,2200(\mathrm{C} \square \mathrm{N}) ;{ }^{1} \mathrm{H}$ NMR: 7.34 (s, 1H, benzylic proton), 7.12-6.92 (m, 6H, Ar- $\mathrm{H}), 6.80$ (s, $2 \mathrm{H}, \mathrm{NH}_{2}$, exchangeable with $\left.\mathrm{D}_{2} \mathrm{O}\right), 4.20$ (s, $1 \mathrm{H}$, pyran-H), 3.82, $3.74\left(2 \mathrm{~s}, 12 \mathrm{H}, 6 \mathrm{OCH}_{3}\right), 3.30(\mathrm{~b}$, $1 \mathrm{H}, \mathrm{CH}-5), 2.50-1.50\left(\mathrm{~m}, 4 \mathrm{H}, 2 \mathrm{CH}_{2}\right.$ of cyclohexene ring), $1.22\left(\mathrm{~d}, 3 \mathrm{H}, 5-\mathrm{CH}_{3}\right) ; \mathrm{MS}(\mathrm{EI}): \mathrm{m} / \mathrm{z} 474\left[\mathrm{M}^{+}\right]$ (100), $337\left[\mathrm{M}^{+}-\mathrm{Ph}\left(\mathrm{OCH}_{3}\right)_{2}\right]$ (65). Anal. Calcd. for $\mathrm{C}_{28} \mathrm{H}_{30} \mathrm{~N}_{2} \mathrm{O}_{5}: \mathrm{C}, 70.86 ; \mathrm{H}, 6.37 ; \mathrm{N}, 5.90 \%$. Found: C, 70.84; H, 6.40; N, 5.88\%.

2-Amino-8-(3,4,5-trimethoxy-benzylidene)-4-(3,4,5trimethoxy-phenyl)-5-methyl-5,6,7,8-tetra-hydro4H-chromene-3-carbonitrile (6c): Yield 87\%, mp $209^{\circ} \mathrm{C}$ (EtOH); IR (KBr, cm $\left.{ }^{-1}\right): 3400,3200,2210 ;{ }^{1} \mathrm{H}$ NMR: 6.92 (s, 1H, benzylic proton), 6.73 (s, 2H, $\mathrm{NH}_{2}$, exchangeable with $\left.\mathrm{D}_{2} \mathrm{O}\right), 6.62,6.40(\mathrm{~s}, 2 \mathrm{H}, \mathrm{Ar}-\mathrm{H}), 3.98$ (s, $1 \mathrm{H}$, pyran-H), 3.83, 3.75, $3.61\left(3 \mathrm{~s}, 18 \mathrm{H}, 6 \mathrm{OCH}_{3}\right)$, 3.28 (b, 1H, CH-5), 1.92-1.54 (2m, 4H, cyclohexene protons), 1.14 (d, 3H, 5- $\left.\mathrm{CH}_{3}\right)$; MS (EI): $\mathrm{m} / \mathrm{z} 534\left[\mathrm{M}^{+}\right]$ (100), $367\left[\mathrm{M}^{+}-\mathrm{Ph}\left(\mathrm{OCH}_{3}\right)_{3}\right]$ (45), 323 [367-CN, $\mathrm{NH}_{2}$, $2 \mathrm{H}]$ (74). Anal. Calcd. For $\mathrm{C}_{30} \mathrm{H}_{34} \mathrm{~N}_{2} \mathrm{O}_{7}$ : C, 67.39; H, $6.41 ; \mathrm{N}, 5.24 \%$. Found: C, 67.35; H, 6.39; N, 5.27\%.

Synthesis of 2-amino-8-(substituted-benzylidene)-4aryl-5-methyl-5,6,7,8-tetrahydro-quinoline-3carbonitrile (7a-c):

Method A: A mixture of compound 5a-c (5 mmol), malononitrile $(0.33 \mathrm{~g}, 5 \mathrm{mmol})$ and ammonium acetate anhydrous ( $2 \mathrm{~g}, 40 \mathrm{mmol})$ in $100 \mathrm{ml}$ ethanol containing on few drops of triethylamine. The reaction mixture was refluxed for $3 \mathrm{~h}$. and then poured onto cold water, the formed solid was filtered off, washed with water, dried and crystallized from the proper solvent to give $7 \mathrm{a}-\mathrm{c}$, respectively.

Method B: A mixture of compound 6a-c $(5 \mathrm{mmol})$, ammonium acetate $(40 \mathrm{mmol})$ and few drops of triethylamine in absolute ethanol $100 \mathrm{~mL}$ was refluxed for $8 \mathrm{~h}$. The solvent was concentrated under reduced pressure, the formed solid was filtered off, dried and crystallized from the proper solvent to give products identified as 7a-c, by their $\mathrm{mp}$ and $\mathrm{R}_{\mathrm{f}}$-values in comparison with authentic samples previously obtained by Method A. 
Am. J. Applied Sci., 8 (10): 945-952, 2011

Table 1: Concentrations resulting in growth inhibition of $50 \%\left(\log _{10} \mathrm{GI}_{50}\right)$ of in vitro human tumor cell lines

\begin{tabular}{|c|c|c|c|c|c|c|c|c|c|c|}
\hline \multirow[b]{2}{*}{ Panel/cell line } & \multicolumn{10}{|c|}{ Compounds } \\
\hline & $4 a$ & $4 \mathrm{~b}$ & $4 \mathrm{c}$ & $5 \mathrm{c}$ & $6 a$ & $6 \mathrm{~b}$ & $6 \mathrm{c}$ & $7 \mathrm{a}$ & $7 b$ & $7 \mathrm{c}$ \\
\hline \multicolumn{11}{|l|}{ Leukemia } \\
\hline CCRF-CEM & -4.7 & -4.8 & -4.0 & -5.9 & -5.3 & -5.6 & -5.7 & -4.6 & -5.4 & -4.6 \\
\hline HL-60(TB) & -4.6 & -4.8 & -4.0 & - & -5.5 & -5.6 & -5.8 & -5.5 & -5.3 & -4.3 \\
\hline $\mathrm{K}-562$ & -4.6 & -4.6 & -4.0 & -5.6 & -5.0 & -5.4 & -5.5 & -5.4 & -4.9 & -4.5 \\
\hline MOLT-4 & -4.7 & -5.6 & -4.5 & - & -5.4 & -5.6 & -5.7 & -4.9 & -5.5 & -4.6 \\
\hline RPMI-8226 & -4.9 & -5.0 & -4.0 & -6.5 & -5.1 & -5.7 & -5.8 & -4.7 & -5.4 & -4.2 \\
\hline SR & -5.0 & -6.6 & -6.9 & - & - & - & -5.8 & -8.0 & - & -4.6 \\
\hline \multicolumn{11}{|c|}{ Non-small cell lung cancer } \\
\hline A549/ATCC & & - & - & -5.6 & - & - & -5.5 & -5.4 & - & -4.0 \\
\hline EKVX & -4.7 & -4.5 & -4.4 & -5.5 & -5.3 & -5.7 & -5.5 & -4.1 & -5.9 & -4.5 \\
\hline HOP-62 & -4.7 & -4.8 & -4.9 & -5.4 & -5.7 & - & -5.1 & -4.2 & -5.7 & -4.0 \\
\hline HOP-92 & - & - & - & -4.0 & - & - & -5.5 & - & - & -4.2 \\
\hline NCI-H226 & -4.7 & -4.0 & -4.2 & - & -5.0 & -5.6 & -5.5 & -4.2 & -5.2 & - \\
\hline NCI-H23 & -4.5 & -4.0 & -4.0 & - & -4.9 & -5.6 & -5.4 & -5.4 & -5.3 & - \\
\hline NCI-H322M & -4.2 & -4.1 & -4.0 & -5.0 & -4.9 & -5.4 & -5.4 & -4.0 & -4.5 & -4.0 \\
\hline NCI-H460 & -4.5 & - & - & - & - & -5.5 & -5.4 & & - & - \\
\hline NCI-H522 & - & - & - & - & - & - & -5.6 & -4.5 & - & - \\
\hline \multicolumn{11}{|l|}{ Colon cancer } \\
\hline COLO 205 & -4.6 & -4.4 & -4.0 & -5.6 & -4.9 & -5.6 & -5.7 & -4.4 & -5.2 & - \\
\hline HCC-2998 & - & - & - & - & - & - & -5.4 & -4.6 & - & - \\
\hline HCT-116 & -4.7 & -4.0 & -4.0 & -5.5 & -4.9 & -5.5 & -5.7 & -4.1 & -5.1 & -4.0 \\
\hline HCT-15 & -4.5 & -4.4 & -4.0 & -5.7 & -5.0 & -5.5 & -5.7 & -5.1 & -5.2 & -4.6 \\
\hline HT-29 & -4.5 & -4.4 & -4.0 & -5.5 & -5.2 & -5.6 & -5.7 & -4.5 & -5.2 & -4.5 \\
\hline KM12 & -4.7 & -4.2 & -4.0 & -5.6 & -4.9 & -5.5 & -5.4 & -4.4 & -5.1 & -4.3 \\
\hline SW-620 & -4.2 & -4.0 & -4.0 & -5.4 & -4.7 & -5.4 & -5.4 & -4.3 & -4.0 & -4.1 \\
\hline \multicolumn{11}{|c|}{ Central nervous system } \\
\hline SF-268 & -4.2 & -4.0 & -4.0 & -5.5 & -4.9 & -5.0 & -5.4 & - & -4.3 & -4.6 \\
\hline SF-295 & - & -4.9 & -4.5 & - & - & -5.5 & -5.4 & -4.4 & -5.2 & - \\
\hline SF-539 & -4.6 & -4.5 & -4.9 & - & -4.8 & -5.8 & -5.6 & -4.3 & -4.6 & - \\
\hline SNB-19 & -4.2 & -4.0 & -4.1 & -5.3 & -4.7 & -5.0 & -4.9 & -4.5 & -4.1 & -4.3 \\
\hline SNB-75 & -4.9 & -5.8 & -5.0 & -4.7 & - & -5.6 & -5.9 & -4.2 & - & -6.8 \\
\hline U251 & -4.4 & -4.4 & -4.0 & -5.3 & -4.8 & -5.4 & -5.5 & -4.8 & -5.0 & -4.3 \\
\hline \multicolumn{11}{|l|}{ Melanoma } \\
\hline LOX IMVI & -4.6 & -4.4 & -4.0 & -5.5 & -4.9 & -5.5 & -5.7 & -4.0 & -4.2 & -4.4 \\
\hline MALME-3M & -4.6 & -4.5 & -4.4 & - & -4.8 & -5.6 & -5.6 & -4.9 & -4.8 & - \\
\hline M14 & -4.5 & -4.2 & -4.0 & -5.7 & -4.8 & -5.5 & -5.7 & -4.2 & -4.5 & -4.3 \\
\hline SK-MEL-2 & -4.7 & -4.0 & -4.1 & -5.8 & -5.0 & -5.7 & -5.7 & -4.2 & -5.6 & -4.0 \\
\hline SK-MEL-28 & -4.5 & -4.0 & -4.0 & -5.4 & -4.8 & -5.4 & -5.5 & -4.3 & -4.3 & -4.0 \\
\hline SK-MEL-5 & -4.7 & -4.6 & -4.0 & -5.7 & -4.8 & -5.7 & -5.6 & -4.4 & -5.3 & -4.1 \\
\hline UACC- 257 & -4.6 & -4.4 & -4.0 & -5.4 & -4.5 & -5.4 & -5.4 & -4.5 & -5.7 & -4.0 \\
\hline UACC-62 & -4.7 & -4.3 & -4.0 & -5.7 & -4.6 & -5.5 & -5.6 & -4.7 & -4.1 & -4.0 \\
\hline \multicolumn{11}{|c|}{ Ovarian cancer } \\
\hline IGROV1 & -4.7 & -4.8 & -4.3 & -5.6 & -4.9 & -5.6 & -5.3 & -4.2 & -4.8 & -6.0 \\
\hline OVCAR-3 & -4.5 & -4.5 & -4.0 & - & -4.9 & -5.4 & -5.4 & -4.3 & -5.0 & - \\
\hline OVCAR-4 & - & - & - & - & - & - & -5.5 & -4.0 & - & -4.5 \\
\hline OVCAR-5 & -4.4 & -4.0 & -4.0 & -5.5 & -4.8 & -5.6 & -5.2 & -4.0 & -4.4 & -4.0 \\
\hline OVCAR-8 & -4.6 & -4.1 & -4.2 & -5.6 & -4.9 & -5.4 & -5.6 & -4.2 & -5.0 & -4.9 \\
\hline SK-OV-3 & -4.6 & -4.1 & -4.1 & -5.0 & -4.7 & -5.5 & -5.3 & -4.0 & -4.7 & -4.0 \\
\hline \multicolumn{11}{|l|}{ Renal cancer } \\
\hline $786-0$ & -4.4 & -4.1 & -4.4 & -5.4 & -4.9 & -5.5 & -5.7 & -4.0 & -5.1 & -4.4 \\
\hline A498 & -4.5 & - & -4.0 & -5.8 & - & -5.4 & -5.4 & -4.6 & - & - \\
\hline ACHN & -4.4 & -4.5 & -4.0 & -5.5 & -4.8 & -5.4 & -5.4 & -4.4 & -4.8 & -4.0 \\
\hline CAKI-1 & -4.2 & -4.0 & -4.0 & -5.5 & -4.8 & -5.4 & -5.4 & -4.7 & -4.2 & -4.0 \\
\hline RXF-393 & -4.6 & -4.0 & -4.7 & -5.6 & -4.8 & -5.8 & -5.8 & - & -5.4 & -4.1 \\
\hline SN12C & -4.4 & -4.4 & -4.0 & -5.4 & -4.9 & -5.5 & -5.5 & -4.0 & -4.0 & -4.4 \\
\hline TK-10 & -4.4 & -4.0 & -4.0 & -5.6 & -4.6 & -5.4 & -5.4 & -4.3 & -5.0 & -4.1 \\
\hline UO-31 & -4.7 & -5.3 & -4.0 & -5.4 & -5.0 & -5.6 & -5.4 & -4.6 & -5.5 & - \\
\hline \multicolumn{11}{|c|}{ Prostate cancer } \\
\hline PC-3 & -4.7 & -4.4 & -4.1 & -5.6 & -4.9 & -5.5 & -5.7 & -4.9 & -5.5 & -4.3 \\
\hline DU-145 & -4.4 & -4.0 & -4.0 & -5.1 & -4.8 & -5.3 & -5.3 & - & -4.7 & -4.3 \\
\hline \multicolumn{11}{|l|}{ Breast cancer } \\
\hline MCF7 & -4.5 & -4.4 & -4.0 & -5.5 & -4.9 & -5.5 & -5.6 & -4.6 & -5.2 & -4.5 \\
\hline
\end{tabular}


Am. J. Applied Sci., 8 (10): 945-952, 2011

\begin{tabular}{|c|c|c|c|c|c|c|c|c|c|c|}
\hline NCI-ADR-RES & -4.6 & - & -4.0 & - & -4.9 & -5.6 & -5.4 & -4.3 & -5.2 & - \\
\hline MDA-MB-231/ATCC & -4.0 & -4.2 & -4.5 & -5.1 & -5.0 & -5.5 & -5.6 & -4.0 & -5.0 & -4.4 \\
\hline HS 578T & -4.5 & -4.1 & -4.3 & -5.1 & -4.8 & -5.4 & -5.7 & - & -5.0 & -4.7 \\
\hline MDA-MB-435 & -4.5 & - & -4.0 & -5.5 & -4.7 & -5.6 & -5.6 & -4.6 & -5.0 & -4.0 \\
\hline MDA-N & -4.6 & -4.0 & -4.0 & -5.5 & -4.8 & -5.5 & -5.6 & -4.1 & -4.5 & -4.0 \\
\hline BT-549 & -4.7 & -4.2 & -4.0 & -6.0 & -4.8 & -5.5 & -5.2 & -4.1 & -5.1 & - \\
\hline T-47D & -5.7 & -5.5 & -5.7 & -5.0 & -6.4 & -5.7 & -5.7 & -4.3 & -5.9 & - \\
\hline
\end{tabular}

2-Amino-8-(p-chlorobenzylidene)-4-(pchlorophenyl)-5-methyl-5,6,7,8-tetrahydroquinoline-3-carbonitrile (7a): Yield $83 \%$ [A], 60\% [B], mp $23222^{\circ} \mathrm{C}(\mathrm{MeOH})$; IR $\left(\mathrm{KBr}, \mathrm{cm}^{-1}\right): 3500,3300$, 2200; ${ }^{1} \mathrm{H}$ NMR: 7.71 (s, 1H, benzylic proton), 7.58-7.14 (m, $8 \mathrm{H}, \mathrm{ArH}), 6.52\left(\mathrm{~s}, 2 \mathrm{H}, \mathrm{NH}_{2}\right.$, exchangeable with $\left.\mathrm{D}_{2} \mathrm{O}\right), 4.03(\mathrm{~s}, 1 \mathrm{H}$, pyran-H), $3.34(\mathrm{~b}, 1 \mathrm{H}, \mathrm{CH}-5), 2.98$ (b, $\left.1 \mathrm{H}, \mathrm{CH}-\mathrm{CH}_{3}\right), 1.91-1.43(2 \mathrm{~m}, 4 \mathrm{H}$, cyclohexene protons), 1.03 (d, 3H, 5- $\left.\mathrm{CH}_{3}\right) ; \mathrm{MS}(\mathrm{EI}): \mathrm{m} / \mathrm{z} 420\left[\mathrm{M}^{+}\right]$ (100), $405\left[\mathrm{M}^{+}-\mathrm{CH}_{3}\right](64), 368[404-\mathrm{HCl}](65), 316$ [468-2CN] (44). Anal. Calcd. for $\mathrm{C}_{24} \mathrm{H}_{19} \mathrm{Cl}_{2} \mathrm{~N}_{3}$ : C, 68.57; H, 4.56; Cl, 16.87; N, 10.00\%. Found: C, 68.53; $\mathrm{H}, 4.51 ; \mathrm{Cl}, 16.78 ; \mathrm{N}, 9.96 \%$.

2-Amino-8-(3,4-dimethoxybenzylidene)-4-(3,4dimethoxyphenyl)-5-methyl-5,6,7,8-tetrahydroquinoline-3-carbonitrile (7b): Yield 95\% [A], 60\% [B], mp $192^{\circ} \mathrm{C}(\mathrm{EtOH}) ; \mathrm{IR}\left(\mathrm{KBr}, \mathrm{cm}^{-1}\right): 3500,3200$, 2200; ${ }^{1} \mathrm{H}$ NMR: 7.71 (s, 1H, benzylic proton), 7.10-6.60 $(\mathrm{m}, 6 \mathrm{H}, \mathrm{Ar}-\mathrm{H}), 6.50\left(\mathrm{~s}, 2 \mathrm{H}, \mathrm{NH}_{2}\right.$, exchangeable with $\left.\mathrm{D}_{2} \mathrm{O}\right), 3.82,3.76,3.64,3.55\left(4 \mathrm{~s}, 12 \mathrm{H}, 4 \mathrm{OCH}_{3}\right), 2.90(\mathrm{~m}$, $1 \mathrm{H}, \mathrm{CH}-5), 1.80-1.40(\mathrm{~m}, 4 \mathrm{H}$, cyclohexene protons), 0.90 (d, 3H, 5- $\left.\mathrm{CH}_{3}\right) ; \mathrm{MS}(\mathrm{EI}): \mathrm{m} / \mathrm{z} 471\left[\mathrm{M}^{+}\right](100), 440$ $\left[\mathrm{M}^{+}-\mathrm{OCH}_{3}\right](64), 425\left[440-\mathrm{CH}_{3}\right](45), 308[425-\mathrm{HCN}$, $\left.3 \mathrm{OCH}_{3}\right]$ (64). Anal. Calcd. for $\mathrm{C}_{28} \mathrm{H}_{29} \mathrm{~N}_{3} \mathrm{O}_{4}$ : C, 71.31; H, 6.19; N, 8.91\%. Found: C, 71.28; H, 6.17; N, 8.87\%.

2-Amino-8-(3,4,5-trimethoxy-benzylidene)-4-(3,4,5trimethoxy-phenyl)-5-methyl-5,6,7,8-

tetrahydroquinoline-3-carbonitrile (7c): Yield 90\% [A], $74 \%[\mathrm{~B}] ; \mathrm{mp} 230^{\circ} \mathrm{C}(\mathrm{MeOH})$; IR $\left(\mathrm{KBr}, \mathrm{cm}^{-1}\right)$ : 3500, 3300, 2210; ${ }^{1} \mathrm{H}$ NMR: 7.48 (s, $1 \mathrm{H}$, benzylic proton), $6.54\left(\mathrm{~s}, 2 \mathrm{H}, \mathrm{NH}_{2}\right.$, exchangeable with $\mathrm{D}_{2} \mathrm{O}$ ), $6.50(\mathrm{~s}, 4 \mathrm{H}, \mathrm{Ar}-\mathrm{H}), 3.90,3.80,3.70,3.60(4 \mathrm{~s}, 18 \mathrm{H}$, $\left.6 \mathrm{OCH}_{3}\right), 3.24(\mathrm{~m}, 1 \mathrm{H}, \mathrm{CH}-5), 1.90-1.50(\mathrm{~m}, 4 \mathrm{H}$, cyclohexene protons), 1.05 (d, 3H, 5- $\left.\mathrm{CH}_{3}\right)$; MS (EI): $\mathrm{m} / \mathrm{z} 531\left[\mathrm{M}^{+}\right](100), 338\left[\mathrm{M}^{+}\right.$-trimethoxybenzylidene] (57), $215\left[282-3 \mathrm{OCH}_{3}, \mathrm{NH}\right]$ (74). Anal. Calcd. for $\mathrm{C}_{30} \mathrm{H}_{33} \mathrm{~N}_{3} \mathrm{O}_{6}: \mathrm{C}, 67.77 ; \mathrm{H}, 6.25 ; \mathrm{N}, 7.90 \%$. Found: $\mathrm{C}$, $67.74 ; \mathrm{H}, 6.28 ; \mathrm{N}, 7.86 \%$.

Anticancer activity: Some of the synthesized compounds were selected and screened for their anticancer activity. Each compound was tested at five different concentrations against 60 cell lines of nine types of human cancers, namely, leukemia, lung, colon,
CNS, melanoma, ovarian, renal, prostate and breast cancer. Results are expressed as $\log _{10} \mathrm{GI}_{50}$, which the drug concentration $(\mathrm{M})$ is causing a $50 \%$ reduction in the net protein increase in control cells during the drug incubation (Lomox and Naryanan, 1981) Table 1. Some of the synthesized compounds showed good anticancer activity at low concentration compared with 5fluorodeoxyuridine $\log _{10} \quad \mathrm{GI}_{50}=-4.7$ as reference control.

\section{RESULTS}

In continuation to our search for new heterocyclic chemistry based anticancer, the suggestion, synthesis, structure elucidation of some naphthalene, chromene and quinoline derivatives 3-7 were realized herein using 3-methylcyclohexanone 1 as a starting material. Some of the synthesized compounds were selected and screened for their anticancer activity. Each compound was tested at five different concentrations against 60 cell lines of nine types of human cancers, namely, leukemia, lung, colon, CNS, melanoma, ovarian, renal, prostate and breast cancer. Some of the tested compounds were better exhibited in vitro antitumor activities at low concentration $\left(\log _{10} \mathrm{GI}_{50}=-4.7\right)$ against the used human tumor cell lines. From the in vitro observed data it has been noticed that, the selected compounds $4 \mathrm{a}, 4 \mathrm{~b}, 4 \mathrm{c}, 5 \mathrm{c}, 6 \mathrm{a}, 6 \mathrm{~b}, 6 \mathrm{c}, 7 \mathrm{a}, 7 \mathrm{~b}$ and $7 \mathrm{c}$ seem to be the most active prepared derivatives against all the tested cell lines.

\section{DISCUSSION}

Chemistry: 3-Methylcyclohexanone (1) was condensed with malononitrile in the presence of $\beta$-alanine as a catalyst in refluxing ethanol according to literature procedure (Hammam et al., 2001; 2000) to give the 3amino-2,4-dicyano-4-(3-methyl-cyclohexylidene)-

butyramide-2-ene (3) and ylidene malononitrile 2 are not obtained. Cyclization of 3 with aromatic aldehydes, namely, p-chloro-, 3,4-dimethoxy- or 3,4,5trimethoxybenzaldehyde in the presence of alcoholic potassium hydroxide by stirring at room temperature 
gave compounds $4 \mathrm{a}-\mathrm{c}$, which were directly prepared by condensation of compound 1 with malononitrile and aromatic aldehydes in refluxing ethanolic potassium hydroxide. ${ }^{13} \mathrm{C}$-NMR spectral analysis (decoupled and APT) for compounds $4 \mathrm{a}-\mathrm{c}$, exemplified by $4 \mathrm{c}$ added a conclusive support for the proposed structure. It reveals the presence of two signals at 115.9, $115.7 \mathrm{ppm}$ assignable for the two nonmagnetically equivalent nitrile compounds in addition to the remaining carbon signals of the tetrahydronaphthalene derivatives (4a-c) (Fig. 1).

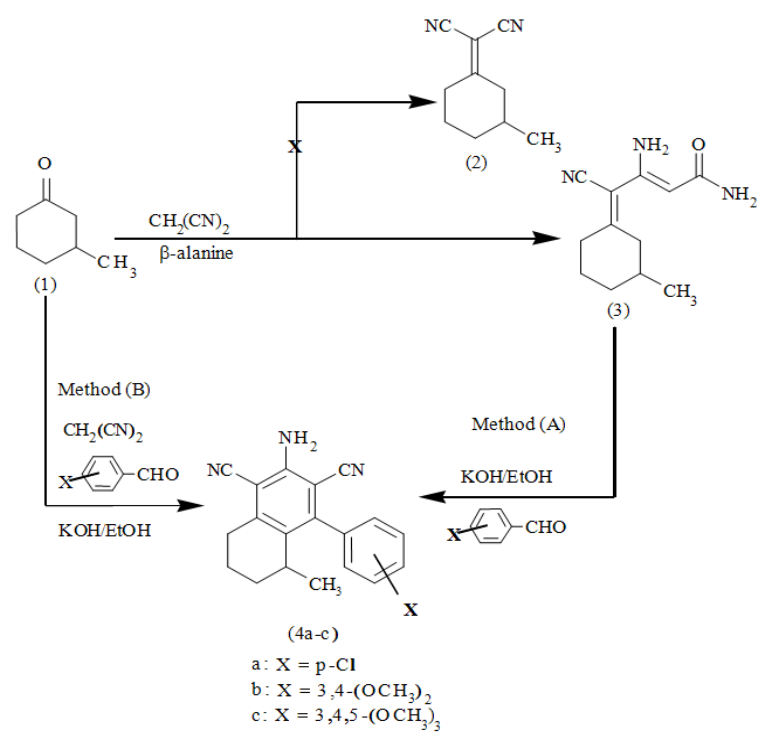

Fig. 1: Synthetic routes for compounds $4 a-c$

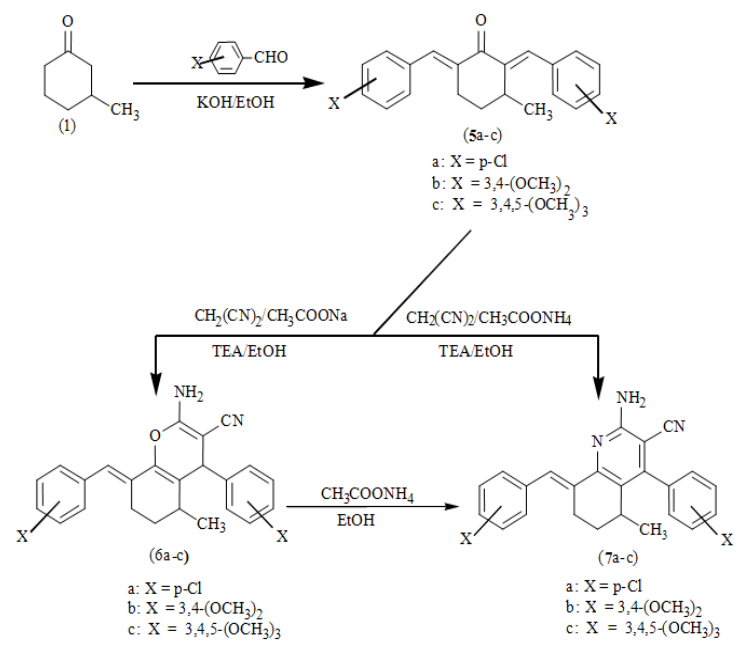

Fig. 2: Synthetic routes for compounds 5a-c-7a-c
Condensation of 1 with aromatic aldehydes, namely, p-chloro-, 3,4-dimethoxy- or 3,4,5-trimethoxybenzaldehyde in ethanolic potassium hydroxide gave the corresponding 2,6-bis-arylmethylene derivatives $5 \mathrm{a}, \mathrm{b}$ and 5c, which were cyclized with malononitrile and sodium acetate or with malononitrile and ammonium acetate in the presence of triethylamine as a catalyst to give chromene-3-carbonitiles (6a-c) and quinoline-3-carbonitiles (7a-c). The later compounds $7 \mathrm{a}-\mathrm{c}$ was prepared by treating of compounds $6 \mathrm{a}-\mathrm{c}$ with ammonium acetate in refluxing ethanol (Fig. 2).

Antitumor screening: Antitumor activity screening for the synthesized compounds utilizing 59 different human tumor cell lines, representing leukemia, melanoma and cancers of the lung, colon, brain, ovary, breast, prostate as well as kidney, was carried out according to the previously reported standard procedure (Xiu et al., 2010; Fylaktakidou et al., 2004; Jung et al., 2005; Ally et al., 1988). The obtained results (Table 1) represent concentrations of the used investigated compounds resulting in growth inhibition of $50 \%\left(\mathrm{GI}_{50}\right)$ for the tested human tumor cell lines. From the in vitro observed data it has been noticed that, the selected compounds 4a, 4b, 4c, 5c, 6a, 6b, 6c, 7a, 7b and 7c seem to be the most active prepared derivatives against all the tested cell lines.

Structural-Activity Relationship (SAR): From the above-obtained results (Table 1), we can conclude that cyanopyridine and pyrane moieties fused to 3methycyclohexane ring are essential for antitumor activities. In the present work, we can suggest that the anticancer activity is due to:

- The presence of nitrogen heterocyclic rings

- The most active compounds being 5c, 6a, 6b, 6c and 7 a against leukemia cell lines

- The presence of the nitrile groups $(\mathrm{CN})$ generally enhancing the activity

- The difference in activity between the compounds which is due to the indicated subsistents in the phenyl group of the molecule

\section{CONCLUSION}

In our previous works, we reported that fused pyrimidine derivatives were proved to be active anticancer agents. In the present work, a series of naphthalene, chromene and quinoline derivatives were synthesized using 3-methylcyclohexanone 1 as a starting material. The antitumor activities of the newly synthesized compounds were evaluated utilizing 60 
different human tumor cell lines, representing leukemia, melanoma, lung, colon, brain, ovary, breast, prostate as well as kidney. Some of the tested compounds were better exhibited in vitro antitumor activities at low concentration $\left(\log _{10} \mathrm{GI}_{50}=-4.7\right)$ against the used human tumor cell lines. From the obtained results, we can conclude that cyanopyridine and pyrane moieties fused to 3-methycyclohexane ring are essential for antitumor activities. In the present work, we can suggest that the anticancer activity is due to the presence of nitrogen heterocyclic rings and the presence of the nitrile groups (CN) generally enhancing the activity.

\section{ACKNOWLEDGMENT}

The researchers extend their appreciation to the Deanship of Scientific Research at King Saud University for funding the work through the research group project No. RGP-VPP-099.

\section{REFERENCES}

Abdel-Latif, A.S. and L.A.M. Abdallah, 2010. Two optimization methods to determine the rate constants of a complex chemical reaction using FORTRAN and MATLAB. Am. J. Applied Sci., 7: 509-517. DOI: 10.3844/ajassp.2010.509.517

Abou-Ghalia, M.H., A.E. Amr and M.M. Abdalah, 2003. Synthesis of some new (NÎt-dipicolinoyl)bis-L-leucyl-DL-norvalyl linear tetra and cyclic octa bridged peptides as new anti-inflammatory agents. Z. Naturforsch., 58: 903-910.

Ally, M.C., D.A. Scudiero, P.A. Monks, M.L. Hursey and M.J. Czerwinski et al., 1988. Feasibility of drug screening with panels of human tumor cell lines using a microculture tetrazolium assay. Cancer Res., 48: 589-601. PMID: 3335022

Amr, A.G.E., 2000. Synthesis of some heterocyclic compounds as potential antimicrobial agents using 2,6-diacetylpyridine as synthon. Ind. J. Heterocycl. Chem., 10: 49-58.

Amr, A.G.E., A.M. Mohamed and A.A. Ibrahim, 2003a. Synthesis of some new chiral tricyclic and macrocyclic pyridine derivatives as antimicrobial agents. Z. Naturforsch., 58: 861-868.

Amr, A.G.E., A.M. Mohamed, S.F. Mohamed, N.A. Agbelhafiz and A.G. Hammam, 2006. Anticancer activities of some newly synthesized pyridine, pyrane, and pyrimidine derivatives. Bioorg. Med. Chem., $\quad 14$ : 5481-5488. DOI: 10.1016/j.bmc.2006.04.045

Amr, A.G.E., M.I. Hegab, A.A. Ibrahim and M.M. Abdalah, 2003b. Synthesis and reactions of some fused oxazinone, pyrimidinone, thiopyrimidinone, and triazinone derivatives with a thiophene ring as analgesic, anticonvulsant, and antiparkinsonian agents. Monatsch. Chem., 134: 1395-1409. DOI: 10.1007/s00706-003-0051-z

Attia, A., O.I.A. Abdel-Salam, A. Amr, I. Stibor and M. Budesinsky, 2000. Synthesis and antimicrobial activity of some new chiral bridged macrocyclic pyridines. Egypt. J. Chem., 43: 187-201.

Attia, A.M.E. and A.E.H.A.A. Ismail, 2003. An approach to acyclo-3-deazapyrimidine Snucleosides via 3,5-dicyano-2(1H)-pyridinethiones. Tetrahedron, 59: 1749-1752. DOI: 10.1016/S00404020(03) 00113-3

Borgio, J.F., B.J. Bency, P.K. Thorat and A.D. Lonkar, 2011. Gynandropsis pentaphylla DC extracts on the production of microbial proteins. Am. J. Drug Discovery Dev., 1: 129-136.

Boyd, M.R. and K.D. Paull, 1995. Some practical considerations and applications of the National Cancer Institute in vitro anticancer drug discovery screen. Drug Dev. Res., 34: 91-109. DOI: 10.1002/ddr.430340203

Bryzgalov, A.O., M.P. Dolgikh, I.V. Sorokina, T.G. Tolstikova and V.F. Sedova et al., 2006. Antiarrhythmic activity of 4,6-di(het)aryl-5-nitro3,4-dihydro-pyrimidin- $(1 \mathrm{H})-2$-ones and its effects on arterial pressure in rats. Bioorg. Med. Chem. Lett., 16 : 1418-1420. DOI: 10.1016/j.bmcl.2005.11.043

Cocco, M.T. C. Congiu, V. Lilliu and V. Onnis, 2005. Synthesis and antiproliferative activity of 2,6dibenzylamino-3,5-dicyanopyridines on human cancer cell lines. Eur. J. Med. Chem., 40: 13651372. DOI: 10.1016/j.ejmech.2005.07.005 PMID: 16137795

Covyeou, J.A., K.E. Atto, C.W. Howard and J.R. Schultz, 2011. Case report of severe injection site reaction associated with intramuscular naltrexone. Am. J. Pharmacol. Toxicol., 6: 1-4. DOI: 10.3844/ajptsp.2011.1.4

Fylaktakidou, C.K., J.D.L. Hadjipavlou, E.K. Litinas and N.D. Nicolaides, 2004. Natural and synthetic coumarin derivatives with anti-inflammatory / antioxidant activities. Curr. Pharm. Des., 10: 38133833. DOI: 10.2174/1381612043382710 PMID: 15579073

Grever, M., S.A. R.Schepartz and B.A. Chabner, 1992. The national cancer institute: Cancer drug discovery and development program. Semin. Oncol., 19: 622-638. PMID: 1462164 
Hammam, A.G., A.F.M Fahmy, A.E. Amr and A.M. Mohamed, 2003. Synthesis of novel tricyclic heterocyclic compounds as potential anticancer agents using chromanone and thiochromanone as synthons. Chem. Inform., 34: DOI: 10.1002/chin.200347130

Hammam, A.G., M.A. Sharaf and N.A. Abdel Hafez, 2001. Synthesis and anti-cancer activity of pyridineand thiazolopyrimidine derivatives using1ethylpiperidone as a synthon. Indian J. Chem., 40B: 213-221.

Hammam, A.G., N.A. Abdel Hafez, W.H. Midura and M. Mikolajczyk, 2000. Chemistry of sevenmembered heterocycles, VI. Synthesis of novel bicyclic heterocyclic compounds as potential anticancer and anti-HIV agents. Z. Naturforsch., 55b: 417-424.

Hassan, S.S.M., M.H. Abou-Ghalia, A.G.E. Amr and A.H.K. Mohamed, 2003a. New lead (II) selective membrane potentiometric sensors based on chiral 2,6-bis-pyridinecarboximide derivatives. Talanta, 60: 81-91. DOI: 10.1016/S0039-9140(03)00038-9

Hassan, S.S.M., M.H. Abou-Ghalia, A.G.E. Amr and A.H.K. Mohamed, 2003b. Novel thiocyanateselective membrane sensors based on di-, tetra-, and hexa-imidepyridine ionophores. Anal. Chem. Acta, 482: 9-18. DOI: 10.1016/S00032670(03)00172-7

Jung, J.C., E.B. Watkins and M.A. Avery, 2005. Synthesis and cyclization reaction of pyrazolin-5one derivatives. Heterocycles, 65: 77-94.

Lomox, N.R. and V.L. Naryanan, 1981. Chemical Structures of Interest to the Division of Cancer Treatment: Compounds in Development: Drugs with Clinical Activity. Pennsylvania State University, pp: 62.

Mohamed, A.M., A.G.E. Amr, M.A. Alsharari, H.R.M. Al-Qalawi and M.O. Germoush et al., 2011. Anticancer activities of some new synthesized thiazolo[3,2-a]pyrido[4,3-d]pyrimidine derivatives. Am. J. Biochem. Biotech., 7: 43-54. DOI: 10.3844/ajbbsp.2011.43.54
Mohamed, S.F., E.M. Flefel, A.E.G. Amr and D.N.A. El-Shafy, 2010. Anti-HSV-1 activity and mechanism of action of some new synthesized substituted pyrimidine, thiopyrimidine and thiazolopyrimidine derivatives. Eur. J. Med. Chem., 45: 1494-1501. DOI: 10.1016/j.ejmech.2009.12.057

Ngoy, J.M., S.E. Iyuke, C.S. Yah and W.E. Neuse, 2011. Kinetic optimization of folic acid polymer conjugates for drug targeting. Am. J. Applied Sci., 8: 508-519. DOI: 10.3844/ajassp.2011.508.519

Quintela, J.M. and C. Peinador, 1996. A ready one-pot preparation for 7-oxa(or thia)-3,4,6triazabenz[d,e]anthracene and 7-oxa-3,4,6,9tetrazabenz[d,e]anthracene derivatives. Tetrahedron, 52: 10497-10506. DOI: 10.1016/0040-4020(96)00570-4

Quintela, J.M. C. Peinador, M.C. Veiga, L.M. Botana, A. Alfonso and R. Riguera, 1998. Synthesis, antihistaminic and cytotoxic activity of pyridothieno- and pyridodithienotriazines. Eur. J. Med. Chem., 33: 887-897. DOI: 10.1016/S02235234(99)80013-0

Quintela, J.M., C. Peinador, L.M. Botana, M. Estevez and R. Riguera, 1997. Synthesis and antihistaminic activity of 2-guanadino-3-cyanopyridines and pyrido[2,3-d]-pyrimidines. Bioorg. Med. Chem., 5: 1543-1553. DOI: 10.1016/S0968-0896(97)00108-9

Rezvani, S. and S. Shariati, 2010. Effect of trehalose Arabidopsis thaliana L. on huntington's disease. Am. J. Plant Physiol., 5: 1-6.

Velusamy, V. and L. Palaniappan, 2011. Compositional analysis $\alpha$-lactalbumin. Am. J. Biochem. Mol. Biol., 1: 106-120.

Xiu, S., A. Shahbazi, L. Wang and C. W. Wallace, 2010. Supercritical ethanol liquefaction of swine manure for bio-oils production. Am. J. Eng. Applied Sci., 3: 494-500. DOI: 10.3844/ajeassp.2010.494.500 